DOI 10.15421/4220014

УДК 539.3

А. С. Каиров, д-р техн. наук, Л. А. Латанская, канд. фриз.-мат. наук, В. А. Каиров, канд. техн. наук

\title{
ЭКСПЕРИМЕНТАЛЬНОЕ ИССЛЕДОВАНИЕ РЕЗОНАНСНЫХ КОЛЕБАНИЙ НЕОДНОРОДНЫХ ЦИЛИНДРИЧЕСКИХ ОБОЛОЧЕК С ОТВЕРСТИЯМИ МЕТОДОМ ГОЛОГРАФИЧЕСКОЙ ИНТЕРФЕРОМЕТРИИ
}

Приведены результаты экспериментальных исследований частот и форм собственных колебаний неоднородных цилиндрических оболочек с отверстиями. Исследования выполнены методом голографической интерферометрии. Установлено влияние отверстий и других конструктивных особенностей на основные динамические характеристики оболочек. Описана методика проведения эксперимента. Экспериментальные данные сопоставляются с численными результатами, полученными методом конечных элементов.

Ключевые слова: цилиндрическая неоднородная оболочка; отверстия; собственные колебания; подкрепляющие ребра; голографическая интерферометрия; метод конечных элементов.

Введение. Тонкостенные оболочечные элементы конструкций широко применяются в современной технике, что вызывает необходимость исследования их резонансных колебаний и определяет актуальность рассматриваемой задачи. При этом наличие отверстий и других конструктивных неоднородностей оказывает существенное влияние на амплитудно-частотные характеристики оболочек, которые отличаются неоднородными полями распределения напряжений, деформаций и перемещений поверхности, сложностью и многообразием фрорм колебаний.

Наряду с численно-аналитическими методами $[1,4,5,7,11$ - 13, 15] определения частот и форм собственных колебаний оболочек особое значение имеют надежные экспериментальные методы [3, 5, 6, 8 - 10, $12,14]$, которые не только дают реальную картину поведения конструкций под действием динамических нагрузок, но и позволяют оценить границы применимости теоретических моделей.

Колебаниям оболочек с отверстиями посвящено сравнительно небольшое количество публикаций, обзор которых и полученные результаты приведены в $[3-5,7,13]$. В основном рассматривались гладкие шарнирно опертые оболочки с одним отверстием. Анализ данных исследований показывает, что для неоднородных оболочечных систем удается определить с приемлемой точностью только низшие частоты и

() А. С. Каиров, Л. А. Латанская, В. А. Каиров, 2020 
формы колебаний. На практике этого недостаточно, поскольку для тонкостенных конструкций, в силу их повышенной чувствительности к вибрациям и в связи с ростом динамичности действующих нагрузок, предусматривается определение более широкой части спектра собственных частот и форм. Для анализа амплитудных полей поверхностных перемещений и повышения достоверности результатов применительно к задачам резонансных колебаний неоднородных оболочек наиболее эфффективными являются методы голографической интерферометрии $[2,3,6,8-10,12,14]$, которые продолжают развиваться и совершенствоваться, находят новые практические приложения.

Целью данной работы является разработка методики экспериментального исследования с помощью голографической интерферометрии амплитудно-частотных характеристик тонкостенных конструктивно неоднородных цилиндрических оболочек с отверстиями, подкрепляющими ребрами, и анализ полученных результатов.

Изложение основного материала. Исследования резонансных колебаний проводились для консольно закрепленных тонкостенных цилиндрических оболочек, изготовленных из стали 20X, с двумя диаметрально расположенными отверстиями $36 \times 36$ мм при $\theta_{0}=2 \pi / 3$ рад. Относительные геометрические характеристики моделей составляют: $L / R=2,3 ; R / h=115 ; R=75$ мм, где $R, L, h$ - радиус, длина и толщина оболочки, $\theta_{\text {о }}$ - угол в радианах между центрами отверстий.

Собственные частоты и формы колебаний определялись в режиме резонансного возбуждения методом голографической интерферометрии, который при установившихся гармонических колебаниях позволяет регистрировать распределения виброперемещений поверхности оболочки при амплитуде порядка длины световой волны. Эксперименты выполнялись по методике, изложенной в $[6,12]$ на вибростенде, функциональная схема которого представлена на рис. 1. Оптическая схема вибростенда аналогична [12].

Модель оболочки 7 устанавливалась в специальном зажимном устройстве 6, реализующем необходимые условия закрепления контура. Гармонические колебания возбуждались контактным пьезоэлектрическим вибратором 8, жестко связанным с зажимным устройством. Частоты вынужденных колебаний регулировались в диапазоне 20...20000 Ги звуковым генератором 11 при варьировании силы тока переменной частоты в электрической цепи вибратора через низкочастотный виброусилитель 9. Для этого звуковой генератор формировал электрические синусоидальные импульсы перестраиваемой длительности с регулируемой задержкой.

Состояние резонанса определялось двухлучевым осциллографом 4, на горизонтальный вход которого подавались электрические сигналы со звукового генератора 11, а на вертикальный вход - электрические сигналы, генерируемые индукционным датчиком 5, пропорциональные величине амплитуды колебаний модели и совпадающие по фазе с ее 
виброперемещениями. Частоты колебаний измерялись электронным частотомером 10 с точностью 1 Ги.

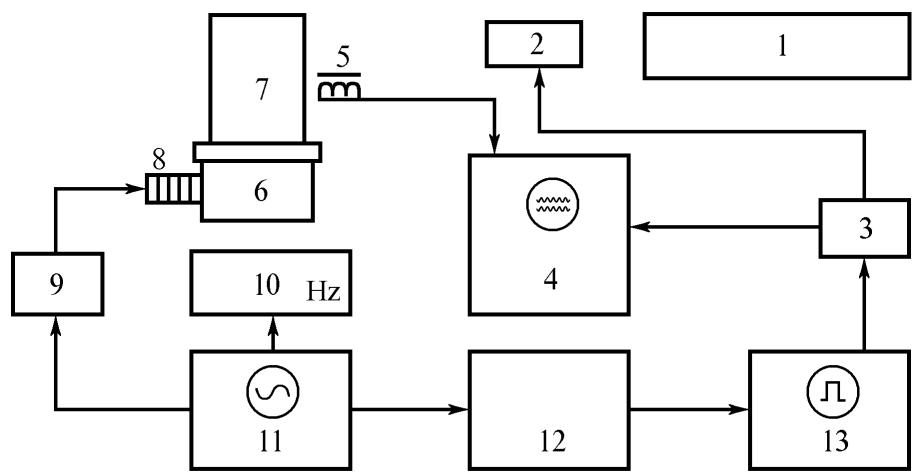

Рис. 1 - Функциональная схема вибростенда:

1 - гелий-неоновый оптический лазер; 2 - электрооптический модулятор;

3 - высокочастотный усилитель; 4 - двухлучевой осциллограф;

5 - индукционный датчик; 6 - зажимное устройство; 7 - модель оболочки;

8 - контактный вибратор; 9 - низкочастотный виброусилитель;

10 - частотомер; 11 - звуковой генератор; 12 - фазовращатель;

13 - импульсный генератор

Момент наступления резонанса также контролировался визуально по установившейся системе интерференционных полос при наблюдении колеблющейся модели через опорную голограмму в реальном времени, что исключало пропуски частот и форм резонансных колебаний. Формы колебаний, соответствующие резонансным частотам и характеризующиеся в окружном направлении числом окружных волн $n$, а в осевом направлении совокупностью полуволн $m$, определялись $c$ помощью осциллографа путем сканирования количества узловых линий между знакопеременными радиальными и продольными амплитудными прогибами оболочки при перемещении индукционного датчика 5 параллельно ее поверхности вдоль линий главных кривизн.

Интерферограммы резонансных форм колебаний регистрировались на заданной частоте методом голографической интерферометрии усреднения по времени с использованием двухлучевой оптической схемы [2, 3, 9, 14]. Наблюдаемые на фотоизображении интерференционные полосы указывают границы полей виброперемещений поверхности модели равных амплитуд пропорционально периоду времени существования каждого из них. Наружные светлые зоны соответствуют неподвижным участкам колеблющейся поверхности.

С целью повышения контрастности интерферограмм для последующей их расшифровки применялось стробирование генерируемого луча лазера 1 электрооптическим модулятором 2 [3, 6, 9, 12]. Для синхронизации стробоимпульсов с определенной фазой колебаний оболочки 
электрический сигнал звукового генератора 11 через фразовращатель 12 поступал на импульсный генератор 13, генерирующий прямоугольные импульсы, и после усиления высокочастотным усилителем 3 подавался на электрооптический модулятор 2. Частота стробирования задавалась кратной частоте колебаний модели. Контроль синхронизации стробоимпульсов с частотой колебаний модели осуществлялся двухлучевым осциллографом 4. Уровень возбуждения подбирался таким, чтобы амплитудные прогибы не превышали 1/20 толщины оболочки и обеспечивалась нормальная разрешимость интерференционных полос на интерферограммах.

Результаты исследований. С использованием изложенной методики голографической интерферометрии выполнено исследование влияния квадратных вырезов и подкрепляющих элементов на нижний и средний диапазоны спектра частот и фрорм резонансных колебаний консольно закрепленных цилиндрических оболочек.

Зависимость нижней части спектра частот $f$ от волновых чисел $n$ при фиксированном значении $m=1$ для исследуемых моделей с двумя центральными отверстиями, расположенными симметрично относительно торцов, приведена на рис. 2 , где $f$ - частота колебаний; $n-$ количество волн в окружном направлении; $m$ - количество полуволн вдоль образующей.

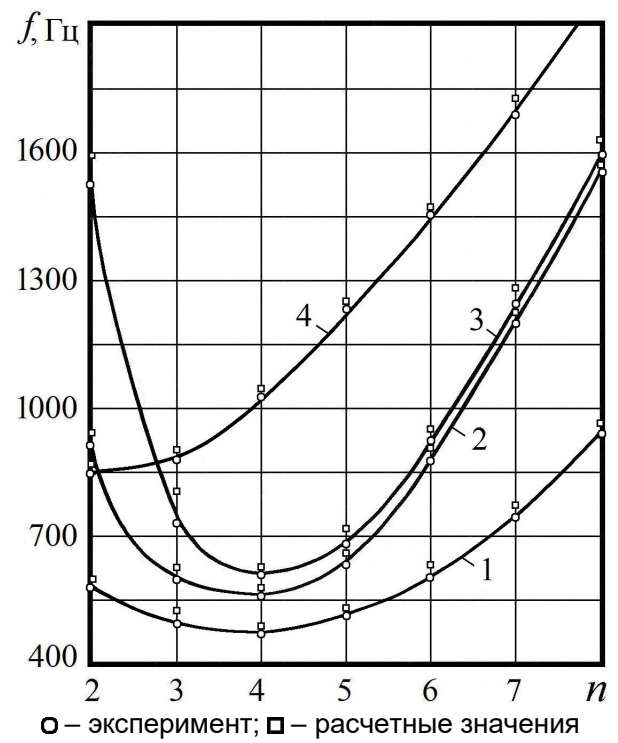

Рис. 2 - Зависимость частот собственных колебаний от волновых чисел п 
Кривая 1 соответствует сплошной оболочке без вырезов, кривая 2 оболочке с двумя квадратными отверстиями $36 \times 36$ мм со свободными кромками, кривая 3 - оболочке с подкрепленными кромками отверстий ребрами прямоугольного сечения $4 \times 1$ мм, кривая 4 - оболочке с подкрепленными отверстиями и шпангоутом $7 \times 1$ мм в верхнем торце. Численные значения частот определялись методом конечных элементов [7]. При этом отверстия, как всякая неоднородность, вызывают расщепление спектра собственных колебаний, полученных в результате расчетов, на парные частоты, для которых формы колебаний совпадают по общей изменяемости, но сдвинуты одна относительно другой в окружном направлении на угол $\varphi=\pi / 2 n$. Большинство форм колебаний нижней части спектра представляет собой простые формы, то есть описываются соответственно функциями $\cos (n x)$ или $\sin (n x)$, за исключением окрестности отверстия.

Из приведенных результатов видно, что отверстия со свободными кромками даже сравнительно небольших размеров приводят к понижению спектра частот, и особенно низшей частоты. При этом частоты располагаются настолько плотно, что фрормы колебаний при незначительном плавном изменении частоты переходят одна в другую и происходит наложение различных формообразований.

Подкрепление контуров отверстий увеличивает локальную жесткость конструкции и приводит к повышению спектра частот колебаний до значений, соответствующих однородным сплошным оболочкам. При этом плотность нижней части спектра снижается, а также уменьшаются амплитуды виброперемещений вдоль контуров отверстий.

При подкреплении верхнего торца оболочки шпангоутом влияние вырезов на частоты колебаний сказывается значительно меньше.

Интерферограммы характерных низших резонансных форм колебаний исследуемых оболочек представлены на рис. 3.

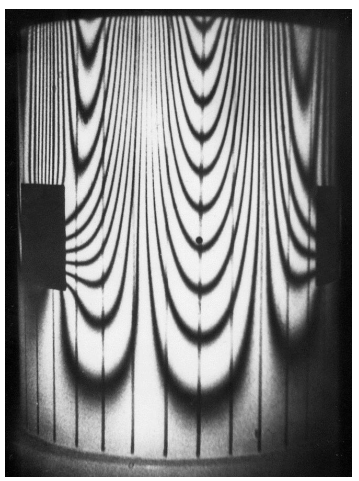

a)

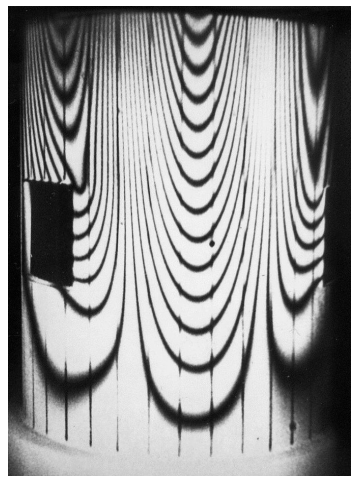

б)

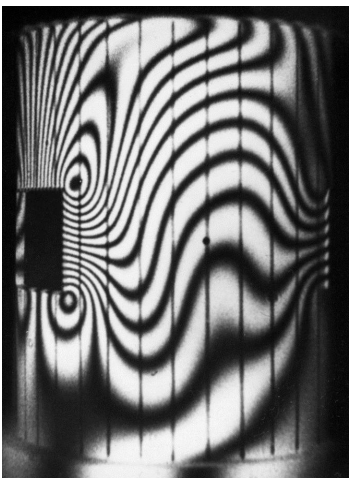

B)

Рис. 3 - Низшие резонансные формы колебаний оболочек с отверстиями 
Особенностью приведенных формообразований является локальная концентрация узловых линий в окрестности отверстий. Это хорошо видно на интерферограмме основной формы колебаний $(n=4)$ оболочки с неподкрепленными отверстиями на низшей частоте 465 Ги (рис. 3,a). На представленном изображении четко выделяются колебания верхней части модели и в углах отверстий, которые являются концентраторами напряжений. Амплитуды колебаний узловых линий равны нулю, а точки поверхности, расположенные по обеим сторонам узловых линий, совершают колебания в противофазе. Данное формообразование отличается от низшей формы колебаний сплошной однородной оболочки [12] и характеризуется локализацией перемещений поверхности в области вырезов. Наибольшие прогибы образуются вдоль кромок отверстия, что согласуется с результатами, полученными для оболочек с различными профилями отверстий $[3,7]$. Формы колебаний при удалении от отверстия носят регулярный характер, свойственный оболочкам без вырезов. Понижение низшей собственной частоты вызвано локальными колебаниями участков поверхности у краев отверстия. На средних частотах и в верхней части спектра образуются сложные формы колебаний, характерной особенностью которых является повышенная изменяемость перемещений поверхности модели в зоне расположения отверстия. Экспериментальные результаты, в отличие от расчетных, не содержат сопряженных форм.

Подкрепление контуров отверстий ребрами локализует амплитуды полей виброперемещений. Низшая форма колебаний оболочки с частотой 517 Ги $(n=4)$ представлена на рис. 3,б.

Интерферограмма основной формы колебаний оболочки с подкрепленными контурами отверстий и шпангоутом, расположенным в верхнем торце, приведена на рис.3,в. На ней наблюдается характерная структура прогибов поверхности. Оболочка колеблется на частоте 848 Гц с волновыми числами $n=2$ и $m=1$. Влияние отверстий на формообразование более локализовано.

Анализ полученных результатов показывает, что отверстия создают локальную инерционную неоднородность оболочечных конструкций, оказывая существенное влияние на их основные динамические характеристики, распределение внутренних напряжений и деформаций, что вызывает необходимость осуществления мероприятий по отстройке от нежелательных резонансов. При подкреплении отверстий и оболочек ребрами жесткости резонансные частоты значительно возрастают, а волновые числа в окружном направлении уменьшаются, внося локальные искажения в амплитудные поля виброперемещений поверхности и изменяя формы колебаний, что подтверждается результатами [7].

Полученные результаты в целом соответствуют известным экспериментальным и теоретическим данным для неподкрепленных оболочек $[3,4]$. Характеристики резонансных колебаний, полученные экспериментально (рис. 2), достаточно хорошо согласуются с результатами расчета конструкции методом конечных элементов. Расчетные и экспе- 
риментальные значения собственных частот для большинства фрорм колебаний отличаются меньше чем на 6\%, а сами формы, включая высокочастотные, совпадают по параметру $n$.

Выводы. Разработан эффективный подход к исследованию резонансных колебаний тонкостенных оболочечных конструкций с помощью голографической интерферометрии. Получены новые экспериментальные результаты, характеризующие влияние отверстий и подкрепляющих ребер на собственные частоты и формы колебаний конструктивно неоднородных цилиндрических оболочек вращения. Результаты работы могут быть полезными при разработке новых расчетных моделей для модального анализа неоднородных оболочек с отверстиями.

\section{БИБЛИОГРАФИЧЕСКИЕ ССЫЛКИ}

1. Басов К.А. ANSYS: Справочник пользователя. М.: ДМК Пресс, 2005. 640 с.

2. Весm Ч. Голографическая интерферометрия. М.: Мир, 1982. 504 с.

3. Гудрамович В.С.., Дзюба А.П., Селиванов Ю.М. Методы голографической интерферометрии в механике неоднородных тонкостенных конструкций: монографія. Д.: Лира, 2017. 288 с.

4. Гузь А.Н., Чернышенко И.С., Чехов В.Н. Теория тонких оболочек, ослабленных отверстиями / Методы расчета оболочек: в 5 т. Т.1. К.: Наукова думка, 1980. 636 с.

5. Заруцкий В.A. О комплексных экспериментальных исследованиях устойчивости и колебаний конструктивно-неоднородных оболочек // Прикл. механика. 2001. Т. 37, № 8. С. 38-67.

6. Каиров А.С., Латанская Л.А., Каиров В.А. Экспериментальное исследование свободных колебаний подкрепленных цилиндрических оболочек с присоединенными твердыми телами // Проблеми обчислювальної механіки і міцності конструкцій: зб. наук. праць. Д.: ІМА-прес, 2009. Вип 13. С. 107-113.

7. Качров А.С., Латанская Л.А., Каиров В.А. Собственные колебания ребристых цилиндрических оболочек с отверстиями // Проблеми обчислювальної механіки і міцності конструкцій: зб. наук. праць. Д.: Ліра, 2019. Вип. 30. С. 96-104.

8. Ларіонова О.О., Рожковський В.Ф., Сохач Ю.В. Голографічні технології в авіаційно-космічній техніці: навч. посіб./ під ред. В. П. Малайчука. Д.: ДНУ, 2003. 272 с.

9. Островский Ю.И., Щепинов В.П., Яковлев В.В. Голографические интерференционные методы измерения деформаций. М.: Наука, 1988. 248 с.

10. Экспериментальная механика: в 2 кн. Кн.1 / под. ред. А. Кабаяси; пер. с англ. М.: Мир, 1990. 616 с.

11. Bathe K.J. Numerical methods in finite element analysis. Englewood Cliffs: Prentice-Hall. 2006. 564 p.

12. Kairov A.S., Zolotoi Y.G. Determination of the vibration displacements and stresses in the vibrations of structurally inhomogeneous shells using the method of holographic interferometry // Jour. of mathematical sciences. 1998. Vol. 92, No. 5. P. 4193-4195.

13. Kairov A.S. Effect of holes of the eigenmodes of reinforced shells of rotations // Journal of Mathematical Sciences. 2001. Vol. 103, No. 3. P. 393-397.

14. Kreis T. Handbook of Holographic Interferometry: Optical and Digital Methods. New York: WILEY-VCH Verlag Gmbh \& Co, 2005. 542 p.

15. Zienkiewich $\boldsymbol{O}$. C., Teylor R. L. The finite element method for solid and structural mechanics. New York: Elsevier, 2005. 632 p. 
О. С. Каіров, д-р техн. наук, Л. О. Латанська, канд. фріз.-мат. наук, В. О. Каіров, канд. техн. наук

\title{
ЕКСПЕРИМЕНТАЛЬНЕ ДОСЛІДЖЕННЯ РЕЗОНАНСНИХ КОЛИВАНЬ НЕОДНОРІДНИХ ЦИЛІНДРИЧНИХ ОБОЛОНОК 3 ОТВОРАМИ МЕТОДОМ ГОЛОГРАФІЧНОЇ ІНТЕРФЕРОМЕТРІЇ
}

\begin{abstract}
Наведено результати експериментальних досліджень частот і форм власних коливань неоднорідних циліндричних оболонок 3 отворами. Дослідження виконані методом голографічної інтерферометрії. Встановлено вплив отворів та інших конструктивних особливостей на основні динамічні характеристики оболонок. Описана методика проведення експерименту. Експериментальні дані порівнюються з числовими результатами, отриманими методом скінченних елементів.
\end{abstract}

Ключові слова: циліндрична неоднорідна оболонка; власні коливання; отвори; підкріплюючі ребра; голографрічна інтерферометрія; метод скінченних елементів.

Тонкостінні оболонкові елементи конструкцій широко використовуються у сучасній техніці, що викликає необхідність дослідження їх резонансних коливань і визначає актуальність даної задачі. Наявність отворів та інших конструктивних неоднорідностей оказує істотний вплив на амплітудно-частотні характеристики оболонок, які відрізняються неоднорідними полями розподілу напружень, деформацій і переміщень поверхні, складністю і різноманіттям форм коливань.

Разом з чисельно-аналітичними методами $[1,4,5,7,11$ - 13, 15] визначення частот і форм власних коливань особливе значення мають надійні експериментальні методи [3, 5, 6,8 - 10, 12, 14], які не тільки дають реальну картину поведінки конструкцій під дією динамічних навантажень, але і дозволяють оцінити межі застосовності теоретичних моделей.

Коливанням оболонок з отворами присвячено порівняно невелика кількість публікацій, огляд яких та отримані результати наведені в [3 - 5, $7,13]$. В основному розглядалися гладкі шарнірно обперті оболонки 3 одним отвором. Аналіз досліджень показує, що для неоднорідних оболонкових систем вдається визначити з прийнятною точністю тільки нижчі частоти і форми коливань. Для аналізу амплітудних полів поверхневих переміщень і підвищення достовірності результатів стосовно до задач резонансних коливань неоднорідних оболонок найбільш ефективними є методи голографічної інтерферометрії [2, 3, 6, 8 - 10, 12, 14], які продовжують розвиватися і вдосконалюватися, знаходять нові практичні застосування.

Метою даної роботи є розробка методики експериментального дослідження за допомогою голографрічної інтерферометрії амплітудночастотних характеристик тонкостінних конструктивно неоднорідних циліндричних оболонок з отворами, підкріплювальними ребрами та аналіз отриманих результатів. 


\section{A. S. Kairov, Dr. Sci. (Tech.), L. A. Latanskaya, PhD (Phys.-Math), V. A. Kairov, PhD (Tech.) \\ EXPERIMENTAL INVESTIGATION OF RESONANT VIBRATIONS OF NON-HOMOGENEOUS CYLINDRICAL SHELLS WITH HOLES BY HOLOGRAPHIC INTERFEROMETRY}

The results of the experimental studies of natural frequencies and waveforms of natural vibrations of non-homogeneous cylindrical shells with holes are presented. The investigations have been carried out by holographic interferometry method. The influence of holes and other design features on the main dynamic characteristics of shells is established. The method of conducting the experiment is described. The experimental data are compared with the numerical results obtained by the finite element method.

Keywords: non-homogeneous cylindrical shell; free vibrations; holes; reinforced ribs; holographic interferometry; finite element method.

The non-homogeneous shells constructions with cutouts are widespread in modern technique. Therefore, the problems which deal with resonant vibrations of the structurally non-homogeneous shells are very relevant actual. To determine the amplitude-frequency characteristics, together with numerical and analytical methods, experimental methods are used, which are of particular importance. They give a real picture of the behavior of thin-walled constructions under the influence of dynamic loads and allow us to assess the limits of applicability of computational models.

Relatively few publications are devoted to the vibrations of shells with holes, a review of which and the results obtained are given in [3-5, 10]. Analysis of the research data shows that for non-homogeneous shell systems it is possible to determine with acceptable accuracy only the lowest frequencies and forms of natural vibrations. In practice, this is not enough, since it is necessary to know a wider part of the spectrum of natural frequencies and waveforms. Holographic interferometry methods [2, 3, 6-8, 11] are the most effective for analyzing the amplitude fields of displacements due to their high accuracy and increasing the reliability of the results of resonant vibrations.

The main aim of this work is to investigate the natural frequencies and waveforms of vibration of the cylindrical shell with holes and stiffening ribs by holographic interferometry and to compare the results obtained with the calculations performed.

The investigations were performed by holographic interferometry method. The method of conducting the experiment is developed. The interference patterns of the waveforms of the research object and new dependences of the influence of its structural non-homogeneity on the resonant vibrations were obtained. It is found that the presence of cutouts, reinforcing ribs and their discrete arrangement create a local inertial inhomogeneity and significantly affect the amplitude-frequency characteristics of the structure. Such dependencies have a great practical value. The experimental data are compared with the numerical results obtained by the finite element method and show good convergence. 


\section{REFERENCES}

1. Basov K. A. ANSIS: User reference. Moscow: DMK Press, 2005. 640 p. (in Russian).

2. West Ch. Holographic Interferometry. Moscow: Mir, 1982. 504 p. (in Russian).

3. Gudramovych V. S., Dzyuba A. P., Selivanov Yu. M. Methods of holographic interferometry in the mechanics of heterogeneous thin-walled structures. Dnepr: Lyra, 2017. 288 p. (in Russian).

4. Guz A. N., Chernyshenko I. S., Chekhov V. N. Theory of thin shells weakened by holes / Methods of calculation of shells: In 5 vol. Vol.1. Kyiv: Naukova dumka, 1980. 636 p. (in Russian).

5. Zarutckiy V. A. The constructional non-homogeneuos shells stability and oscillations experimental complex // Applied mechanics. 2001. Vol. 37, № 8. P.38-67. (in Russian).

6. Kairov A. S., Latanskaya L. A., Kairov V. A. Experimental study of free vibrations of the reinforced cylindrical shells with attached solid bodies // Problems of Computational Mechanics and Strength of Structures: Col. of sci. art. 2009. Vol. 13. P. 107-113. (in Russian).

7. Kairov A. S., Latanskaya L. A., Kairov V. A. Natural vibrations of ribbed cylindrical shells with cutouts // Problems of Computational Mechanics and Strength of Structures: Col. of sci. art. 2019. Vol. 30. P. 96-104. (in Russian).

8. Larionova O. O., Rozhkovsky V. F., Sohach Yu. V. Holographic technologies in aerospace engineering: textbook. Tool / Ed. V. P. Malaychuk. Dnepropetrovsk: RVV DNU, 2003. 272 p. (in Ukrainian).

9. Ostrovsky Yu. I., Shchepinov V. P., Yakovlev V. V. Holographic interference methods of deformation measurement. Moscow: Nauka. 248. p. (in Russian).

10. Experimental mechanics: In 2 vol. Vol.1/ Ed. A. Kabayashi. Moscow: Mir, 1990. 616 p. (in Russian)

11. Bathe K. J. Numerical methods in finite element analysis. Englewood Cliffs: Prentice-Hall. 2006. 564 p.

12. Kairov A. S., Zolotoi Y. G. Determination of the vibration displacements and stresses in the vibrations of structurally inhomogeneous shells using the method of holographic interferometry // Jour. of mathematical sciences. 1998. Vol. 92, No. 5. P. 41934195.

13. Kairov A. S. Effect of holes of the eigenmodes of reinforced shells of rotations // Journal of Mathematical Sciences. 2001. Vol. 103, No. 3. P. 393-397.

14. Kreis T. Handbook of Holographic Interferometry: Optical and Digital Methods. New York: WILEY-VCH Verlag Gmbh \& Co, 2005. 542 p.

15. Zienkiewich O. C., Teylor R. L. The finite element method for solid and structural mechanics. New York: Elsevier, 2005. 632 p.

Национальный университет кораблестроения имени адмирала Макарова Николаев, Украина

Надійшла до редколегії 19.10.2020 